\title{
Two-stage hepatectomy (R0) with portal vein ligation- towards curing patients with extended bilobular colorectal liver metastases
}

\author{
K. Homayounfar • T. Liersch • G. Schuetze • \\ M. Niessner • A. Goralczyk • J. Meller • C. Langer • \\ B. M. Ghadimi • H. Becker • T. Lorf
}

Accepted: 27 November 2008 /Published online: 16 December 2008

(C) The Author(s) 2008. This article is published with open access at Springerlink.com

\begin{abstract}
Background and aims Patients with bilobular colorectal liver metastases (CRLM) experience poor prognosis, especially when curative resection cannot be achieved. However, resectability in these patients is often limited by low future remnant liver volume (FRLV). The latter can be enhanced by a two-stage liver resection, using portal vein ligation to induce liver hypertrophy. The aim of this prospective pilot study was to evaluate safety, secondary resectability, and time to recurrence of two-stage hepatectomy with portal vein ligation (PVL) and complete surgical clearance of the FRLV in patients with bilobular CRLM. Materials and methods Out of 24 patients $(63 \pm 8.26$ years) with extended bilobular CRLM (metachronous $n=10$, synchronous $n=14$ ), 18 received preoperative 5-FU-based chemotherapy combined with oxaliplatin or irinotecan. Staging included thoracoabdominal computed tomography
\end{abstract}

K. Homayounfar and T. Liersch contributed equally to this manuscript and study.

K. Homayounfar $(\bowtie) \cdot$ T. Liersch • M. Niessner · A. Goralczyk •

C. Langer $\cdot$ B. M. Ghadimi $\cdot$ H. Becker $\cdot$ T. Lorf

Department of General and Visceral Surgery,

University Medical Center Göttingen, Georg-August-University,

Robert-Koch-Str. 40,

37075 Göttingen, Germany

e-mail: khomayounfar@chirurgie-goettingen.de

G. Schuetze

Department of Diagnostic Radiology,

University Medical Center Göttingen,

Göttingen, Germany

J. Meller

Department of Nuclear Medicine,

University Medical Center Göttingen,

Göttingen, Germany and ${ }^{18}$ F-fluorodeoxyglucose-positron emission tomography scans. First-stage procedure consisted of PVL, resection of all CRLM in the FRLV, and radiofrequency ablation (RFA) of CRLM situated near the future resection plane.

Results During first-stage procedure, $7 \times$ RFA, $4 \times$ nonanatomical resections, and $4 \times$ bisegmentectomies were performed additionally to PVL. FRLV/body-weight ratio increased from $0.4 \%$ to $0.6 \%$ within 55 days (median) after PVL. Second-stage hepatectomy was performed in 19 patients without tumor progression. R0 resection was possible in 14 patients. During a median follow-up of 17 months, intrahepatic recurrence occurred in two, and extrahepatic recurrence in nine out of 14 patients.

Conclusion Two-stage hepatectomy with PVL and complete surgical clearance of FRLV is safe even after intensified systemic chemotherapy resulting in a curative resection rate of $58.3 \%$ ( $73.7 \%$ of re-explored cases).

Keywords Bilobular colorectal liver metastases - Two-stage hepatectomy · Portal vein ligation · Insufficient future remnant liver volume $\cdot$ Liver hypertrophy

\section{Introduction}

Liver resection is the only potentially curative treatment option of colorectal cancer metastases (CRLM). It has become the standard of care even in patients with multiple metastases, resulting in 5-year survival rates of $37-50 \%$ in specialized centers $[1,2]$. However, in case of bilobular involvement, patients experience a poorer outcome, with 5year survival rates of only $20 \%$ [3]. As this still is beneficial when compared to intensive systemic chemotherapy alone [4], surgical approaches are recommended even in this 
challenging subgroup of patients, provided that curative resection appears achievable. Unfortunately, $70-80 \%$ of patients presenting with multiple and/or bilobular CRLM are not resectable at the time of diagnosis [5]. In patients without extrahepatic spread, unresectability is mainly due to an insufficient amount of future remnant liver volume (FRLV) [6], which, in the absence of chronic liver disease, can be estimated as less than $30 \%$ of the initial total liver volume [7] or a FRLV to body weight ratio of $<0.5 \%$ [8]. It has been demonstrated that secondary resectability in these cases can be achieved by either preoperative chemotherapy, portal vein occlusion, or both. While preoperative chemotherapy leads to an effective downsizing of CRLM [9], portal vein occlusion, performed as surgical ligation (PVL) or as interventional embolization (PVE), induces significant hypertrophy of the FRLV [10]. Portal vein occlusion thereby allows extended two-stage hepatectomy without transient liver failure [11]. In our experience, neither preoperative chemotherapy nor the number of metastases influence tumor-related overall survival when curative resection can be achieved (Fig. 1a,b). Additionally, Shaw et al. [12] reported 5-year survival rates of $44 \%$ after second hepatectomy for recurrent CRLM. Adam et al. [13] even described a 5 -year survival rate of $32 \%$ after third hepatectomy. Although these data are encouraging and seem to justify aggressive multidisciplinary approaches, there is still no consensus on what the optimal oncological strategy should be.

The present prospective pilot study on 24 patients with bilobular CRLM describes an intensive multimodal treatment concept, including preoperative chemotherapy and two-stage hepatectomy with portal vein ligation (PVL) and complete surgical clearance of the FRLV. The paper focuses on feasibility, safety, and surgical and preliminary oncological outcome.

\section{Materials and methods}

All interventions were performed according to established surgical procedures and actual guidelines for the treatment of colorectal and hepatobiliary diseases by the German Society of Surgeons.

Between April 2005 and November 2007, 24 patients with a mean age of $63 \pm 8.26$ (range 42-76) years and presenting with bilobular CRLM were selected for a twostage liver resection with PVL. All data were collected prospectively. The characteristics of the 24 patients are summarized in Table 1. Seven female and 17 male patients were included in the study. Primary tumor location was colon and rectum in 12 patients each. One patient received neoadjuvant radiochemotherapy before rectal resection, and eight patients were treated by chemotherapy in an adjuvant

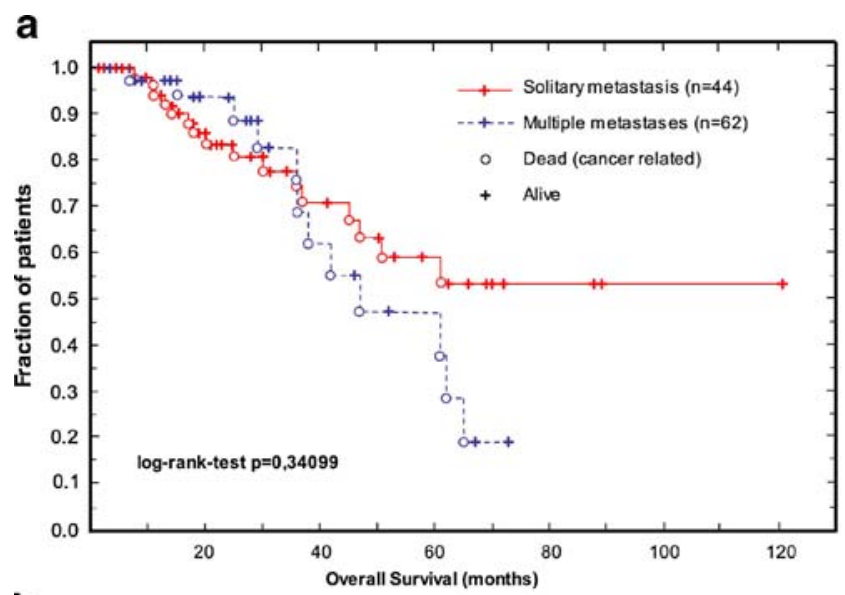

b

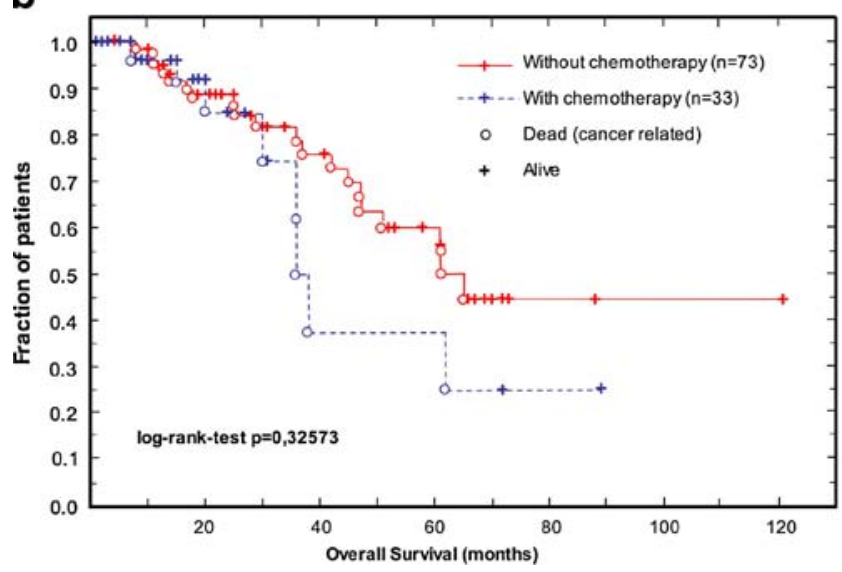

Fig. 1 a Kaplan-Meier-plot on overall survival after salvage resection of colorectal liver metastases with curative intent $(n=106)$. Patients were stratified based on number of metastases but irrespective of preoperative and adjuvant treatment (University Medical Center Goettingen, Department for General and Visceral Surgery 20012007). b Kaplan-Meier plot on overall survival after salvage resection of colorectal liver metastases with curative intent $(n=106)$. Patients were stratified according to preoperative treatment (chemotherapy versus no chemotherapy) irrespective of number of metastases, resection type, or adjuvant treatment (University Medical Center Goettingen, Department of General and Visceral Surgery 2001-2007)

setting after resection of the primary tumor. CRLM were synchronous in 14 patients (time between primary cancer resection and diagnosis of CRLM $<3$ month) and metachronous in ten patients, with a median disease-free survival between primary tumor resection and diagnosis of CRLM of 20 (range, 8-183) months.

Three patients had been included in our study because of intrahepatic recurrence. Two of these had undergone previous curative non-anatomic liver resection. The third patient had been treated by systemic chemotherapy with 5-fuorouracil, folinic acid, and oxaliplatin for synchronous CRLM, resulting in complete remission based on Response Evaluation Criteria in Solid Tumors (RECIST) criteria [14] but presented with intrahepatic recurrence 22 months later. 
Table 1 Patient characteristics

\begin{tabular}{llc}
\hline & Number & Percent \\
\hline Patients & 24 & 100 \\
Sex & & \\
Female & 7 & 29.2 \\
Male & $85.9 \pm 16.8$ & 70.8 \\
Weight (kg) & & \\
Primary cancer & 12 & 50 \\
Colon & 12 & 50 \\
Rectum & & \\
Primary tumor stage & & 0 \\
UICC I & 0 & 20.8 \\
UICC II & 5 & 20.8 \\
UICC III & 5 & 58.4 \\
UICC IV & 14 & \\
Systemic therapy of primary cancer & & 33.4 \\
5FU/FA + RT 50,4 Gy (neoadjuvant) & 1 & \\
5-FU/FA \pm Oxaliplatin (adjuvant) & 8 & 58.4 \\
Detection of hepatic metastases & & 41.6 \\
$\quad$ Synchronous & \\
Metachronous & 14 & \\
\hline
\end{tabular}

UICC Union international contre le cancer, 5-FU 5-fluorouracil, FA folinic acid, $R T$ radiotherapy, $S D$ standard deviation

${ }^{\mathrm{a}}$ Mean $\pm \mathrm{SD}$

${ }^{\mathrm{b}} \mathrm{TNM} / \mathrm{UICC}$ classification (up to $16 \mathrm{~cm}$ above anocutaneous verge as measured by rigide rectoscopy)

${ }^{\mathrm{c}}$ Less than 3 months between resection of primary tumor and diagnosis of intrahepatic metastases

Eighteen (75\%) patients received preoperative chemotherapy with oxaliplatin or irinotecan; six (33\%) of them were treated additionally with the vascular endothelial growth factor antibody, bevacizumab. According to RECIST criteria, eight of the 18 patients $(44.4 \%)$ showed stable disease or even partial response, while in ten patients (55.6\%), progressive disease confined to the liver was observed (Table 2). However, in the latter, resectability had never been assessed by an experienced hepatobiliary surgeon before, but at restaging prior to our study, curative resection seemed to be achievable despite intrahepatic tumor progression. At the time of referral to our institution, the median preoperative Karnofsky performance index was $>80 \%$.

Preoperative evaluation included thoracoabdominal computed tomography (CT) scan with special regard to portal vein anatomy, as well as 3-dimensional volumetry, and also ${ }^{18} \mathrm{~F}$-fluorodeoxyglucose-positron emission tomography (FDG-PET). All data were preoperatively reviewed by a multidisciplinary team (hepatobiliary surgeon, medical and surgical oncologist, radiologist, and specialist in nuclear medicine). Figure 2 shows the design of our pilot study. The aim of the intensive preoperative imaging conducted was to rule out disseminated intrahepatic CRLM, which we considered as contraindication for primary surgical exploration, and to identify extrahepatic tumor spread. The latter was not considered as contraindication to liver resection when curative resection seemed to be achievable simultaneously to first-stage hepatectomy or during prior separate surgery. Patients with unresectable extrahepatic disease, namely with advanced local recurrence, disseminated pulmonary metastases, or peritoneal carcinomatosis were not eligible for this feasibility study.

\section{First-stage hepatectomy}

First-stage hepatectomy, as the key step in our multimodal therapy concept, was performed in all patients. After upper midline laparotomy with right subcostal (J-shaped) extension, complete exploration of the intraperitoneal cavity was performed. The liver was then evaluated with both bimanual palpation and intraoperative ultrasound to identify the intrahepatic metastatic pattern. Portal vein occlusion was performed at the side of main tumor load. To avoid extensive adhesions during second-stage hepatectomy, mobilization of the liver and especially hilar dissection were carried out most conservatively, with as minimal a division of the falciform ligament and diaphragmatic attachments as possible. Using a right lateral approach to the hepatoduodenal ligament, the main portal vein was then

Table 2 First-stage hepatectomy

\begin{tabular}{|c|c|c|}
\hline & Number & Percent \\
\hline \multicolumn{3}{|c|}{ Preoperative chemotherapy for downsizing of CRLM } \\
\hline None & 6 & 25 \\
\hline FOLFOX/FOLFIRI & 12 & 50 \\
\hline FOLFOX/FOLFIRI + Bevacizumab & 6 & 25 \\
\hline \multicolumn{3}{|l|}{ Effect of preoperative chemotherapy ${ }^{a}$} \\
\hline Stable disease/ partial remission & 8 & 33.3 \\
\hline Progressive disease & 10 & 41.7 \\
\hline \multicolumn{3}{|l|}{ Surgical procedures } \\
\hline Right PVL & 23 & 95.8 \\
\hline Left PVL & 1 & 4.2 \\
\hline+ radio frequency ablation & $7^{\mathrm{b}}$ & 29.2 \\
\hline+ non-anatomical resection & 4 & 16.6 \\
\hline + bisegmentectomy segment II/III & 4 & 16.6 \\
\hline+ resection of extrahepatic disease & 1 & 4.2 \\
\hline Hospitalisation (days) $^{\mathrm{c}}$ & $12.3 \pm 3.8(8-28) ; 12$ & \\
\hline Mortality & 0 & 0 \\
\hline Morbidity & 3 & 12.6 \\
\hline Wound infection & 1 & 4.2 \\
\hline Cardiac arrhythmia & 1 & 4.2 \\
\hline Urogenitary infection & 1 & 4.2 \\
\hline
\end{tabular}

CTX chemotherapy, CRLM colorectal liver metastases, PVL portal vein ligation

${ }^{a}$ Relating to intrahepatic disease; based on RECIST-criteria [14]

${ }^{\mathrm{b}}$ In two cases both RFA and non-anatomical resection were performed

${ }^{\mathrm{c}}$ Mean \pm SD (range); Median 
Fig. 2 Algorithm for treatment of bilobular colorectal liver metastases applied in the study. After diagnosing bilobular CRLM, coexistence and extent of extrahepatic disease was evaluated and, if necessary, resected. During first-step hepatectomy, the site of main tumor load was identified with subsequent ipsilateral portal vein ligation or transection. CRLM in the future remnant liver lobe were resected completely if present. Those metastases situated near the future resection plane were treated by RFA to achieve local tumor control. Newly diagnosed extrahepatic disease was resected simultaneously. When restaging after liver hypertrophy showed no progression of disease, secondstage hepatectomy with major liver resection was performed. In case of progressive disease, the remaining CRLM were treated by RFA, followed by chemotherapy in palliative intention

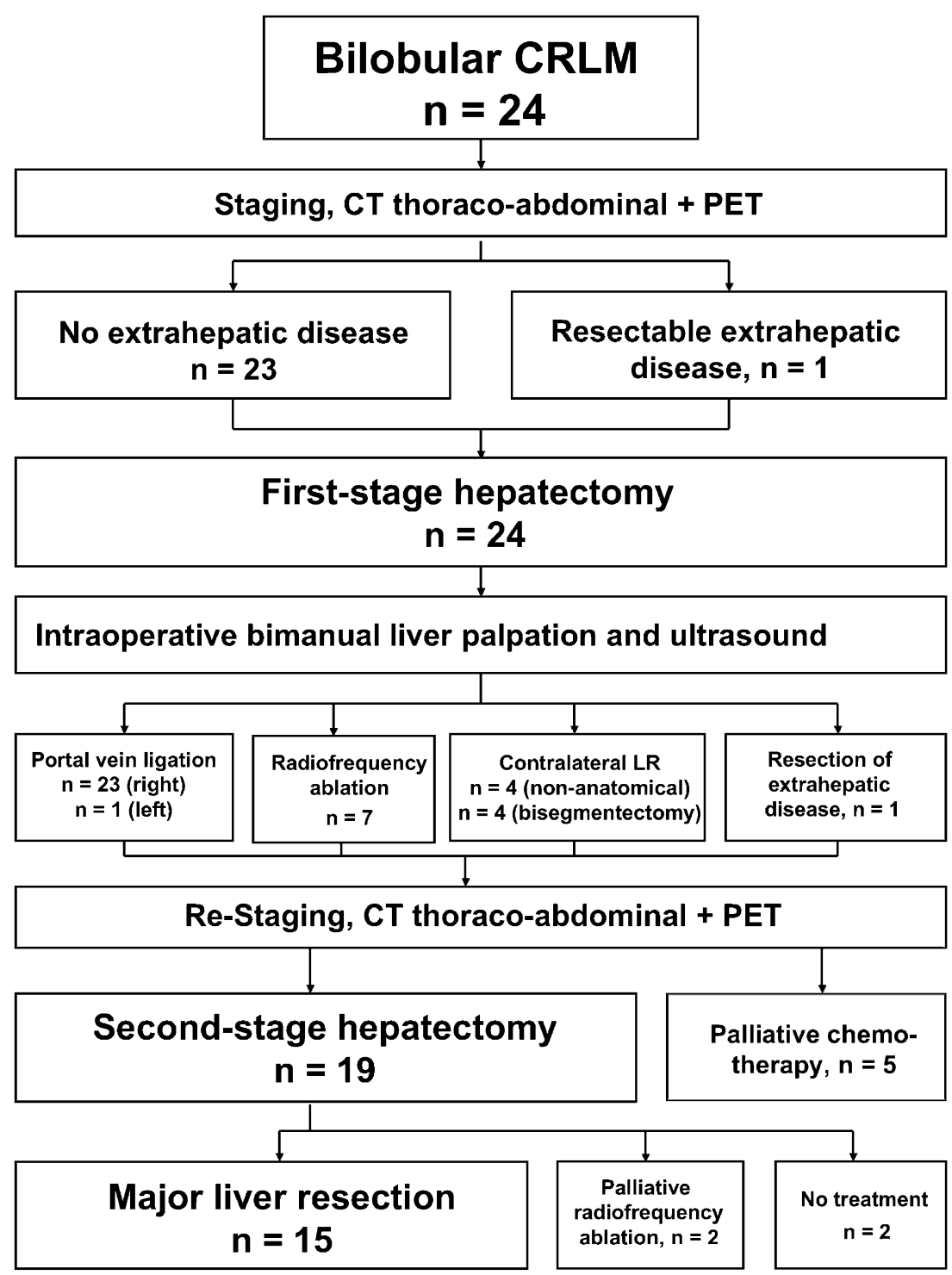

followed until its bifurcation into the left and right portal veins. To facilitate redissection during second-stage hepatectomy, the portal branch selected for occlusion was transected and oversewn using nonabsorbable sutures with the use of vascular clamps. In patients presenting with variant portal vein anatomy, namely a bifurcation of the main portal trunk into a right posterior and right anterior branch with the left portal vein arising from the latter, we modified our technique. A transection of the right posterior branch was performed as described, followed by ligation of the right anterior branch distal of the rise of the left portal vein (Fig. 3). Because CRLM have been reported to grow during liver regeneration following portal vein occlusion $[15,16]$, CRLM situated near the future resection plane were ablated by radiofrequency (RFA), suggesting that growth rate and metastatic potential during hypertrophy could thereby be reduced. This concerned metastases in $\mathrm{S}$ $\mathrm{IVa} / \mathrm{b}$, when right trisegmentectomy was planned, and metastases in segment V/VIII when left trisegmentectomy was planned. CRLM in the lateral segments of the nonligated lobe were not treated by RFA but resected. In cases when only segments I and IV were unaffected by CRLM, right PVL was combined with bisegmentectomy of segment II/III.

Re-evaluation

Re-evaluation was performed routinely 6 weeks after firststage hepatectomy and again consisted of thoracoabdominal CT scan with 3-dimensional volumetry and FDG-PET. All volumetric examinations were reviewed by the same experienced radiologist (G.S.). The second-stage procedure was cancelled when CRLM occurred in the future remnant liver or disseminated extrahepatic tumor spread was diagnosed. No chemotherapy was administered between first-stage and second-stage hepatectomy. 


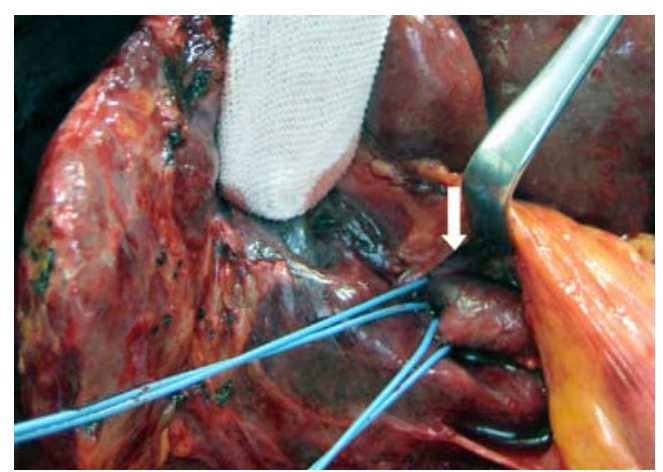

Fig. 3 Intraoperative situs with performed limited lateral dissection of the hepatoduodenal ligament in a patient with variant portal vein anatomy: the first bifurcation of the main portal vein (PV) divides the $\mathrm{PV}$ into a right posterior branch and right anterior branch (both indexed by blue vessel loops), the latter delivering the left portal vein (white arrow). Such anatomical variation is rare occurring in less than $15 \%$ of cases [42]. Transection instead of ligation of the portal vein branches during first-stage procedure results in technically easier complete dissection of the hepatoduodenal ligament during secondstage hepatectomy. It might be difficult to perform transection without liver dissection especially for the right anterior branch in the presence of variant portal vein splitting. Thus, in the displayed situation, we performed transection of the right posterior branch and ligation of the right anterior branch of the portal vein

Second-stage hepatectomy

Depending on the extent of liver resection during the firststage procedure, we performed hemihepatectomy (in cases of previous bisegmentectomy) or trisegmentectomy during the second-stage hepatectomy. Liver parenchyma dissection was carried out without inflow occlusion. Lymph node dissection of the hepatoduodenal ligament was not performed routinely. In patients with resectable extrahepatic disease, this was resected simultaneously even if affecting the lung. According to our treatment paradigm, we cancelled the planned liver resection when new CRLM occurred in the FRLV and treated all detectable CRLM by RFA. The diagnosis of disseminated intra- or extrahepatic tumor spread resulted in termination of the operation. Postoperative liver failure was assessed according to Balzan et al. [17].

Histopathological examination was performed according to standard operating procedures.

\section{Postoperative treatment}

Previous immunohistochemical studies as well as analyses of serum titers of carcinoembryonic antigen (CEA) indicate its expression in $>90 \%$ of colorectal cancer patients [18, 19]. In addition, diagnostic imaging studies with CEAspecific antibodies have shown selective and enhanced localization of colorectal cancer [20, 21]. Therefore, we performed immunohistochemical staining for CEA in all patients, and postoperative radioimmunotherapy with ${ }^{131} \mathrm{I}$ - labetuzumab [22, 23] was indicated on investigators' discretion when staining intensity was assessed as moderate or strong.

Follow-up

Patients were standardly followed up with a physical examination, liver biochemistry, CEA, and abdominal ultrasound every 3 months. Patients consenting to adjuvant radioimmunotherapy underwent thoracoabdominal CT and FDG-PET scans prior to radioimmunotherapy and afterwards, every 6 months. No patient was lost during the follow-up period until June 30, 2008.

\section{Ethical considerations}

All human interventions were performed in accordance with the ethical standards laid down in the 1964 Declaration of Helsinki and have been approved by the regional ethical committee. All persons gave their informed consent prior to their inclusion in the study.

\section{Statistics}

The Kaplan-Meier survival estimates method was used to calculate overall survival, and significant differences were examined using the log-rank test. A difference was considered to be significant when the $p$ value was less than 0.05 . The analysis was performed using the statistical software Statistica (StatSoft, Tulsa, OK, USA).

\section{Results}

First-stage hepatectomy

In $23(95.8 \%)$ of the cases, the right portal vein was ligated or dissected; in one patient, ligation of the portal branches was extended to segment IV. One patient underwent ligation of the left portal vein. Additional treatment to PVL consisted of RFA $(n=7)$, non-anatomical resection $(n=4)$, and bisegmentectomy of the left lateral segments II/III $(n=4)$. In one patient, preoperative CT scan had shown a suspicious, FDG-PET-positive mass ventrocranial of the bladder, which could be identified intraoperatively as a local lymph node recurrence and could be completely resected. The mean hospital stay after first-stage procedure was $12.3 \pm 3.8$ days with no mortality and a morbidity rate of $12.6 \%$ (Table 2 ).

Volumetric analysis

Table 3 gives a summary of the volumetric analysis. The median number of liver metastases detected by CT scan 
Table 3 Volumetric data of the liver parenchyma

\begin{tabular}{lccc}
\hline & Mean \pm SD & Range & Median \\
\hline No. of liver metastases before & $3.1 \pm 2.3$ & $1-7$ & 2 \\
PVL ( $n$ ) & & & \\
Size of largest liver metastasis & $4.3 \pm 2.9$ & $0.9-11$ & 4 \\
before PVL (cm) & & & \\
TLV before PVL (ml) & $1752.3 \pm 413.5$ & 1613.5 \\
FRLV before PVL (ml) & $350.5 \pm 111$ & 322 \\
FRLV/body weight ratio before & $0.42 \pm 0.11$ & & 0.4 \\
PVL & & & \\
No. of liver metastases after & $4.2 \pm 3.3$ & $1-10$ & 4 \\
hypertrophy ( $n$ ) & & & \\
Size of largest liver metastasis & $4.8 \pm 2.9$ & $0.3-11$ & 4.4 \\
after hypertrophy (cm) & & & \\
TLV after hypertrophy (ml) & $1712.4 \pm 397.8$ & 1610 \\
FRLV after hypertrophy (ml) & $475 \pm 171.4$ & 419 \\
time between PVL and re- & $57.9 \pm 18$ & & 55 \\
evaluation (days) & & & \\
$\Delta$ FRLV (ml) & $123.2 \pm 106.7$ & 114 \\
$\Delta$ FRLV (\%) & $35.7 \pm 29.3$ & 36.5 \\
TLV/FRLV ratio & $28 \pm 6.4$ & 29 \\
FRLV/body weight ratio & $0.58 \pm 0.23$ & 0.6 \\
\hline
\end{tabular}

$P V L$ portal vein ligation, $T L V$ total liver volume, $F R L V$ future remnant liver volume

before first-stage procedure was 2.0 (range, 1-7) with a median size of $4.0 \mathrm{~cm}$ (range, 0.9-11) for the largest metastases. Median FRLV had been measured as $322 \mathrm{ml}$ with a FRLV/body weight ratio of 0.4. After PVL, the median number of CRLM increased to 4.0 (range, 1-10), coexistent with a slight increase in the median size of the largest metastasis to 4.4 (range, $0.3-11$ ) $\mathrm{cm}$. While total liver volume remained constant, FRLV increased to $419 \mathrm{ml}$ after $57.9 \pm 18$ days, representing a median volume gain of $36.5 \%$ and a FRLV/body weight ratio of 0.6 before secondstage hepatectomy.

\section{Second-stage hepatectomy}

Second-stage hepatectomy was performed in 19 (79.2\%) of 24 patients (Table 4). Five patients were not eligible for surgical reexploration due to newly diagnosed disseminated pulmonary metastases $(n=2)$ or progression of intrahepatic disease with new metastases in the FRLV $(n=3)$. These five patients received systemic chemotherapy with palliative intent.

Nineteen patients were suitable for re-operation, but liver resection was cancelled in four patients due to occult progressive disease with peritoneal carcinomatosis $(n=2)$, long-segment tumor infiltration of the future remnant left hepatic vein $(n=1)$, or tumor involvement of FRLV $(n=1)$. In the patient presenting with tumor infiltration of the left hepatic vein, curative resection would have been possible by use of extracorporal bypass and vascular reconstruction techniques but was rejected due to an underlying cardiovascular disease. In both patients with intrahepatic tumor progression, CRLM were treated by RFA during the second-stage procedure.

In 14 of 15 cases, the planned hemihepatectomy (after bisegmentectomy during the first-stage procedure (Fig. 4)) or trisegmentectomy could be performed. In one patient, the resection procedure had to be changed from right trisegmentectomy to central liver resection of segment V/VIII because of complete portal vein thrombosis. In two patients, CRLM were adherent to the diaphragm, so its partial resection was necessary to achieve tumor-free margins. In one additional patient, a pulmonary metastasis located in the right lower lobe was simultaneously resected via a transdiaphragmatic approach.

Re-operation was necessary in one case 6 days after curative second-stage hepatectomy due to biliary leakage. However, the patient died 28 days after right trisegmentectomy due to liver failure caused by left hepatic artery thrombosis. Mortality and morbidity rates of the secondstage procedure were $5.3 \%$ and $57.9 \%$, respectively. Based on intensive histopathological examinations, curative resection could be achieved in 14 of 15 (93.3\%) performed

Table 4 Second-stage hepatectomy

\begin{tabular}{|c|c|c|}
\hline & Number & Percent $^{\mathrm{a}}$ \\
\hline Second-stage hepatectomy performed & 19 & $79.2^{\mathrm{b}}$ \\
\hline Liver resection & 15 & $62.5^{\mathrm{b}}$ \\
\hline Right trisegmentectomy (SIV-VIII) & 13 & 68.4 \\
\hline Right hemihepatectomy (SV-VIII) ${ }^{c}$ & 1 & 5.3 \\
\hline Central liver resection (S V/VIII) & 1 & 5.3 \\
\hline Surgical exploration, RFA & 2 & 10.5 \\
\hline Surgical exploration, but no specific therapy & 2 & 10.5 \\
\hline \multicolumn{3}{|l|}{ Multivisceral resection } \\
\hline Diaphragm/ lung & 3 & 15.8 \\
\hline Curative resection rate ${ }^{d}$ & 14 & 93.3 \\
\hline $\begin{array}{l}\text { CEA expression of tumor cells strong or } \\
\text { moderate }^{\mathrm{d}}\end{array}$ & 15 & 100 \\
\hline Mortality & 1 & 5.3 \\
\hline Morbidity ${ }^{\mathrm{f}}$ & 11 & 57.9 \\
\hline Liver insufficiency $^{\mathrm{e}}$ & 3 & 15.8 \\
\hline Biliary leakage & 4 & 21.1 \\
\hline Wound healing & 1 & 5.3 \\
\hline Cardiopulmonal & 4 & 21.1 \\
\hline Reoperation & 1 & 5.3 \\
\hline
\end{tabular}

${ }^{a}$ Percentage in correlation to number of patients re-explored

${ }^{\mathrm{b}}$ Percentage in correlation to number of patients in the study

${ }^{\mathrm{c}}$ After bisegmentectomy S II/III at first-stage hepatectomy

${ }^{\mathrm{d}}$ Only patients with liver resection during second-stage hepatectomy

${ }^{\mathrm{e}}$ Defined by prothrombin time $<50 \%$ and serum bilirubin $>50 \mu \mathrm{mol} /$ 1 on post-op day 5 [17]

${ }^{\mathrm{f}}$ In patients with multiple complications, each is counted separately 


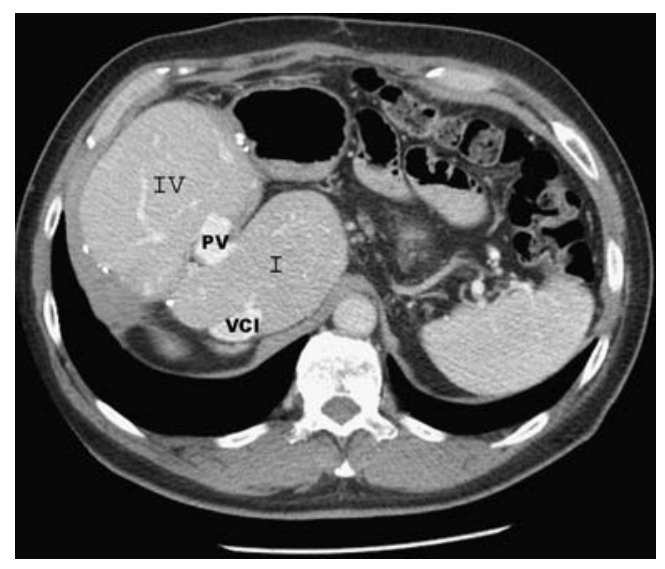

Fig. 4 CT scan of the upper abdomen in portalvenous phase demonstrating enormous hypertrophy of segment I and IV after bisegmentectomy S II/III during first-stage hepatectomy, followed by standard right hemihepatectomy during second-stage hepatectomy. CT scan was performed 18 months after second-stage hepatectomy. $P V$ portal vein, $V C I$ inferior vena cava

liver resections. In all cases, immunohistochemical examination revealed a moderate $(n=1)$ or strong $(n=14)$ expression of CEA on the surface of tumor cells.

\section{Follow-up}

Follow-up was documented for the 13 patients with curative resection. Four patients received no adjuvant therapy following hepatic resection, while eight patients were enrolled in an adjuvant radioimmunotherapy protocol. Two months after R0-resection, one patient died of pulmonary embolism. During a median follow-up period of 17 months, ten patients developed recurrent disease (Table 5) that, at the time of diagnosis, was multifocal in one, confined to the liver in one, and limited to the lung in six patients. Two additional patients presented with bone metastases after a disease-free survival of 5 and 6 months, respectively. Only one patient with a solitary pulmonary metastasis was suitable for surgical therapy and has been free of disease for 22 months, while in all other cases of tumor recurrence, chemotherapy or radiochemotherapy was applied. Three patients with recurrent disease died 14, 17, and 27 months, respectively, after second-stage procedure because of tumor progression. Two other patients with recurrent disease showed no progression under chemotherapy.

\section{Discussion}

This pilot study describes our experience with two-stage hepatectomy using portal vein ligation in patients with bilobular CRLM. These patients are associated with poor prognosis, and only if curative resection can be achieved, 5year survival rates may reach $20 \%$ [3]. Therefore, every attempt should be made to convert initially unresectable to resectable patients which can be achieved by two-stage hepatectomy.

To induce hypertrophy of the FRLV, we favored surgical PVL instead of interventional PVE, although it has been reported by Broering et al. [24] to result in a significantly lower increase in liver volume. However, this conclusion should be restrained because the authors also showed a

Table 5 Follow-up

\begin{tabular}{|c|c|c|c|c|c|c|}
\hline Patient No. & Time to recurrence ${ }^{\mathrm{a}}$ & Recurrence site & Treatment & Outcome at last observation & $\mathrm{OS}^{\mathrm{b}}$ & Status \\
\hline 1 & 4 & Hepar, pulmo & CTX & PD & 14 & DOT \\
\hline $2 \mathrm{P}$ & 1 & Pulmo, osseous ${ }^{\mathrm{c}}$ & CTX & $\mathrm{PD}$ & 27 & DOT \\
\hline 3 & 2 & Pulmo & CTX & $\mathrm{PD}$ & 17 & DOT \\
\hline $4 \mathrm{P}$ & 6 & Pulmo & CTX & PD & 18 & A \\
\hline 5 & 6 & Osseous & RTX & PD & 21 & A \\
\hline 6 & 5 & Osseous & RTX & PD & 10 & A \\
\hline 7 & 2 & Pulmo & CTX & PD & 7 & A \\
\hline 8 & 3 & Hepar, pulmo ${ }^{c}$ & CTX & $\mathrm{SD}$ & 17 & A \\
\hline 9P & 1 & Pulmo & CTX & SD & 17 & A \\
\hline 10 & - & - & - & NED & 2 & $\mathrm{DOC}$ \\
\hline $11 \mathrm{P}$ & - & - & - & NED & 21 & A \\
\hline 12 & - & - & - & NED & 12 & A \\
\hline 13 & 7 & Pulmo & Surgery & NED & 29 & A \\
\hline
\end{tabular}

$C T X$ chemotherapy, $R T X$ radiochemotherapy, $A$ alive, DOT dead of tumor, DOC dead of other cause (pulmonary embolism), $P$ progression under chemotherapy before liver resection, $P D$ progressive disease, $S D$ stable disease, $N E D$ no evidence of disease

${ }^{a}$ Time between second-stage hepatectomy and diagnosis of tumor recurrence (month), last observation 2008, 6, 30

${ }^{\mathrm{b}}$ Time between second-stage hepatectomy and last observation

${ }^{\mathrm{c}}$ Second side of recurrence under chemotherapy 
significantly higher volume gain when portal vein branches to segment IV were occluded simultaneously, and this was done significantly more often in the PVE group. Furthermore, a recent publication by Aussilhou et al. [25] concluded that PVE and PVL result in comparable liver hypertrophy.

Compared to preoperative staging data, the surgical approach with PVL enhances sensitivity and specificity in the detection of CRLM using intraoperative ultrasound [26, 27]. It also allows combined PVL- and CRLM-directed therapy, even simultaneously with primary tumor resection without increasing morbidity or mortality rates [28, 29]. Although not as part of surgery for primary tumor, we performed liver resection up to bisegmentectomy and radiofrequency ablation together with PVL in eight and seven patients, respectively.

A number of studies have compared the efficacy of liver resection and RFA in the treatment of CRLM. They have demonstrated clearly that overall survival rates are higher, and local recurrence rates are lower in patients treated with resection [1, 30, 31]. Moreover, in cases where CRLM could not be resected completely, resection and RFA together did not significantly increase survival compared with RFA alone. Facing these results and remembering the categorical high risk of recurrence in patients with bilobular CRLM, RFA proves inappropriate for the treatment of CRLM in the nonligated hemi-liver.

Accordingly, the present series describes an aggressive two-stage hepatectomy concept with combination of PVL and complete surgical clearance of the nonligated hemiliver. Other authors [6] discussed that PVL combined with liver resection during first-stage hepatectomy may induce postoperative liver failure. In contrast, in our experience with 24 cases, no liver failure due to insufficient amount of liver volume occurred after the first-stage procedure, even though we performed bisegmentectomy of segments II/III in four patients (Table 2). In all patients, we observed a sufficient increase in FRLV using PVL with a mean interval of $57.9 \pm 18$ days, and cancellation of second-stage hepatectomy was in no case based on insufficient hypertrophy.

Addressing oncological strategies, it has to be observed that there is still some controversy about whether chemotherapy, given between the two surgical procedures, is beneficial with regard to the curative resection rate. Severe sinusoidal injury has been observed in liver specimens after chemotherapy prior to liver resection [32-34], with some authors reporting an increased postoperative morbidity [33] - or even mortality rate [34]. Otherwise, intensive systemic chemotherapy with infusional 5-fluorouracil, folinic acid, and oxaliplatin or irinotecan have demonstrated response rates of up to $56 \%$ in primarily unresectable CRLM [35], resulting in curative resection rates of $12.5 \%$ [9], of which a high percentage will ask for two-stage procedures. Tumor progression can be observed during liver hypertrophy not only in the nonligated [15] but also in the ligated lobe [16], which our data also demonstrate with regard to both number and size of CRLM (Table 2). Thus, there is a need for antitumor therapy between first- and second-stage hepatectomy, especially to control micrometastatic disease.

Besides systemic treatment, application of local chemotherapy has been studied in this context. Selzner et al. [36] treated 11 patients with PVL and intrahepatic arterial chemotherapy based on the antimetabolite, floxuridine. Although FRLV increased significantly 3 months after PVL, only six patients showed a partial response [14] to local chemotherapy, with an overall curative resection rate of only $36 \%$. In a recently published study, Lygidakis et al. [28] also combined the surgical approach with three cycles of intra-arterial chemotherapy in 32 patients with synchronous bilobular CRLM. Two days after first-stage procedure including PVL, they started a complex transarterial chemotherapy schedule including oxaliplatin, irinotecan, mitomycin C, 5-fluorouracil, and calcium folinate, as well as the monoclonal antibody, bevacizumab. The chemotherapy was administered over 5 days with the next cycle following after 25 days. Major liver resection was performed 3 weeks after completing the third cycle of chemotherapy. Curative resection was possible in all patients, with a 2-year survival rate of $80 \%$. Considering our number of five patients, though, that did not undergo second-stage hepatectomy based on intrahepatic $(n=3)$ or pulmonary $(n=2)$ progression, plus those four patients without major liver resection during second-stage procedure, a need for further studies on the appropriate management strategy in these patients becomes clear.

Curative resection could be achieved in 14 out of 19 re-explored patients $(73.7 \%)$ even though we included patients with progression under chemotherapy and even patients with intrahepatic recurrence. In one of our patients, complete portal vein thrombosis required a switch in the operative resection strategy. Note that neither tumor progression, prothrombotic disorder, or infection was diagnosed in this case [37]. Instead of right trisegmentectomy, we performed central liver resection with curative intent. Although technically possible, we did not intend central liver resection initially because it is more complex, results in a wide resection plane which increases the risk for biliary fistula, and is associated with a longer parenchymal transection time as well as elevated intraoperative blood loss [38].

Patients with bilobular CRLM which are classified as mT4 according to Gayowski et al. [3] experience poor prognosis when compared to those having unilobular involvement. During early follow-up, we identified lung metastases in four patients already $1(n=2)$ and $2(n=2)$ months after curative liver resection (Table 5), respectively. 
They have to be classified as occult metastatic disease at the time of restaging prior to second-stage hepatectomy. Thus, prolonging the interval between the two surgical procedures to 3 months seems to be reasonable because it would allow occult diseases to be disclosed. However, even though our follow-up period was short, only in two patients was the liver the first site of tumor recurrence, whereas other authors reported an intrahepatic recurrence rate of 48 $75 \%$ during a comparable time frame $[6,39]$. The low intrahepatic recurrence rate may result from our aggressive approach of resecting all CRLM in the FRLV.

Histopathological examination of the obtained liver specimens revealed moderate or even strong expression of carcinoembryonic antigen on the surface of tumor cells in all patients. As a result, postoperative radioimmunotherapy with ${ }^{131}$ I-labetuzumab, as described previously [22, 23], has been offered to all patients resected curatively.

There is interdisciplinary consensus that only unresectable extrahepatic disease, unfit condition, and liver involvement of $>6$ segments excludes patients from liver resection for CRLM [40]. Therefore, the aim of the present study was to evaluate the outcome of consecutive patients fulfilling our inclusion criteria without preselecting them based on previous therapies and their response profiles. The underlying rationale of this study design is the definition of resectability as crucial end point in treatment concepts with curative intent [40]. However, as almost $75 \%$ of patients relapse after liver resection [41] within 2 years postsalvage resection of liver metastases, the observed and expected recurrence rate in our study population with already poor prognosis again argues for aggressive multimodal treatment prior to and especially postsalvage liver resection as well as for the establishment of reliable predictive clinical and molecular parameters that will allow patient stratification.

In conclusion, two-stage hepatectomy with portal vein ligation and complete surgical clearance of FRLV is a reasonable strategy in patients with bilobular, initially unresectable CRLM. The curative resection rate results in $73.7 \%$ of the re-explored cases. Further clinical trials are needed to evaluate whether antitumor therapy during liver hypertrophy may positively affect the curative resection rate by limiting the observed tumor progression after portal vein occlusion. Although not mandatory for volume gain, a second-stage procedure should be delayed by 3-6 months in cases of suspicious lesions found during restaging, in order to reduce unnecessary examinations in patients with unresectable and progressive disease. The described advances in the treatment of patients with bilobular CRLM have radically redefined what constituted an unresectable pattern of CRLM. Any patient with CRLM should be reviewed by a multidisciplinary team of experts, and resectability should be assessed by an experienced hepatobiliary surgeon before a decision on management is made. The treatment of tumor recurrence especially extrahepatic remains a future challenge.

Acknowledgment The authors would like to thank Dr. David M. Goldenberg, Ph.D. for critical comments on the manuscript.

Sources of funding This work was supported by the German Research Society (DFG) and part of the clinical research unit 179 (KFO 179: Biological basis of individual tumor response in patients with rectal cancer), subproject 4.

Open Access This article is distributed under the terms of the Creative Commons Attribution Noncommercial License which permits any noncommercial use, distribution, and reproduction in any medium, provided the original author(s) and source are credited.

\section{References}

1. Abdalla E, Vauthey JN, Ellis LM, Ellis V, Pollock R, Broglio KR et al (2004) Recurrence and outcomes following hepatic resection, radiofrequency ablation, and combined resection/ablation for colorectal liver metastases. Ann Surg 239:818-827

2. Fong Y, Fortner J, Sun RL, Brennan MF, Blumgart LH (1999) Clinical score for predicting recurrence after hepatic resection for metastatic colorectal cancer: analysis of 1001 consecutive cases. Ann Surg 230:309-318

3. Gayowski TJ, Iwatsuki S, Madariaga JR, Selby R, Todo S, Irish $\mathrm{W}$ et al (1994) Experience in hepatic resection for metastatic colorectal cancer: analysis of clinical and pathologic risk factors. Surgery 116:703-710

4. Hurwitz H, Fehrenbacher L, Novotny W, Cartwright T, Hainsworth J, Heim W et al (2004) Bevacizumab plus irinotecan, fluorouracil, and leucovorin for metastatic colorectal cancer. $\mathrm{N}$ Engl J Med 350:2335-2342

5. Adam R (2003) Chemotherapy and surgery: new perspectives on the treatment of unresectable liver metastases. Ann Oncol 14(S2): ii13-16

6. Jaeck D, Oussoultzoglou E, Rosso E, Greget M, Weber JC, Bachellier P (2004) A Two-stage hepatectomy procedure combined with portal vein embolization to achieve curative resection for initially unresectable multiple and bilobular colorectal liver metastases. Ann Surg 240:1037-1051

7. Gruttadauria S, Vasta F, Mivervini MI, Piazza T, Arcadipane A, Marcos A et al (2005) Significance of the effective remnant liver volume in major hepatectomies. Am Surg 71:235-240

8. Truant S, Oberlin O, Sergent G, Lebuffe G, Gambiez L, Ernst O et al (2007) Remnant liver volume to body weight ratio $>0.5 \%$ : a new cut-off to estimate postoperative risks after extended resection in noncirrhotic liver. J Am Coll Surg 204:22-33

9. Adam R, Delvart V, Pascal G, Azoulay D, Delvart V, Paule B et al (2004) Rescue surgery for unresectable colorectal liver metastases downstaged by chemotherapy: a model to predict long-term survival. Ann Surg 240(6):644-657

10. Rous P, Larimore LD (1920) Relation of the portal blood to liver maintenance. J Exp Med 31:609-632

11. Azoulay D, Castaing D, Smail A, Adam R, Cailliez V, Laurent A et al (2000) Resection of nonresectable liver metastases from colorectal cancer after percutaneous portal vein embolization. Ann Surg 231(4):480-486

12. Shaw IM, Rees M, Welsh FKS, Bygrave S, John TG (2006) Repeat hepatic resection for recurrent colorectal liver metastases is 
associated with favourable long-term-survival. Br J Surg 93:457464

13. Adam R, Pascal G, Azoulay D, Tanaka K, Castaing D, Bismuth H (2003) Liver resection for colorectal metastases: the third hepatectomy. Ann Surg 238(6):871-883

14. Therasse P, Arbuck SG, Eisenhauer EA, Wanders J, Kaplan RS, Rubinstein L et al (2000) New guidelines to evaluate the response to treatment in solid tumours. European Organization for Research and Treatment of Cancer, National Cancer Institute of the United States, National Cancer Institute of Canada. J Natl Cancer Inst 92 (3):205-216

15. Elias D, de Baere T, Roche A, Ducreux M, Leclere J, Lasser P (1999) During liver regeneration following right portal embolization the growth rate of liver metastases is more rapid than that of the liver parenchyma. Br J Surg 86:784-788

16. Kokudo N, Tada K, Seki M, Ohta H, Azekura K, Ueno M et al (2001) Proliferative acitivity of intrahepatic colorectal metastases after preoperative hemihepatic portal vein embolization. Hepatology 34:267-272

17. Balzan S, Belghiti J, Farges O, Ogata S, Sauvanet A, Delefosse D, Durand F (2005) The "50-50 criteria" on postoperative day 5: an accurate predictor of liver failure and death after hepatectomy. Ann Surg 242:824-829

18. Goldenberg DM, Sharkey RM, Primus FJ (1976) Carcinoembryonic antigen in histopathology: immunoperoxidase staining of conventional tissue sections. J Natl Cancer Inst 57:11-22

19. Gold P, Goldenberg NA (1997) The carcinoembryonic antigen (CEA): past present, and future. McGill J Med 3:46-66

20. Goldenberg DM, Kim EE, DeLand FH, Bennett S, Primus FJ (1980) Radioimmunodetection of cancer with radioactive antibodies to carcinoembryonic antigen. Cancer Res 40:2984-2992

21. Moffat FL Jr, Pinsky CM, Hammershaimb L, Petrelli NJ, Patt YZ, Whaley FS et al (1996) Clinical utility of external immunoscintigraphy with the IMMU-4 technetium-99 $\mathrm{m} \mathrm{Fab}$ ' antibody fragment in patients undergoing surgery for carcinoma of the colon and rectum: results of a pivotal, phase III trial. The Immunomedics Study Group. J Clin Oncol 14(8):2295-2305

22. Liersch T, Meller J, Kulle B, Behr TM, Markus P, Langer C et al (2005) Phase II trial of carcinoembryonic antigen radioimmunotherapy with 131I-labetuzumab after salvage resection of colorectal metastases in the liver: five-year safety and efficacy results. J Clin Oncol 23(27):6763-6770

23. Liersch T, Meller J, Bittrich M, Kulle B, Becker H, Goldenberg DM (2007) Update of carcinoembryonic antigen radioimmunotherapy with (131)i-labetuzumab after salvage resection of colorectal liver metastases: comparison of outcome to a contemporaneous control group. Ann Surg Oncol 14:2577-2590

24. Broering DC, Hillert C, Krupski G, Fischer L, Mueller L, Achilles EG et al (2002) Portal vein embolization versus portal vein ligation for induction of hypertrophy of the future liver remnant. J Gastrointest Surg 6(6):905-913

25. Aussilhou B, Lesurtel M, Sauvanet A, Farges O, Dokmak S, Goasguen N et al (2007) Right portal vein ligation is as efficient as portal vein embolization to induce hypertrophy of the left liver remnant. J Gastrointest Surg 12(2):297-303 (Epub 2007 Nov 30)

26. Scaife CL, Ng CS, Ellis LM, Vauthey JN, Charnsangavej C, Curley SA (2006) Accuracy of preoperative imaging of hepatic tumours with helical computed tomography. Ann Surg Oncol 13:542-546

27. Wildi SM, Gubler C, Hany T, Petrowsky H, Clavien PA, Jochum $\mathrm{W}$ et al (2007) Intraoperative sonography in patients with colorectal cancer and resectable liver metastases on preoperative FDG-PET-CT. J Clin Ultrasound 36(1):20-26

28. Lygidakis NJ, Bhagat AD, Vrachnos P, Grigorakos L (2007) Challenges in everyday surgical practice: synchronous bilobular hepatic colorectal metastases - newer multimodality approach. Hepatogastroenterology 54:1020-1024

29. Martin R, Paty P, Fong Y, Grace A, Cohen A, DeMatteo R et al (2003) Simultaneous liver and colorectal resections are safe for synchronous colorectal liver metastasis. J Am Coll Surg 197 (2):233-241

30. van Duijnhoven FH, Jansen MC, Junggeburt JM, van Hillegersberg R, Rijken AM, van Coevorden F et al (2006) Factors influencing the local failure rate of radiofrequency ablation of colorectal liver metastases. Ann Surg Oncol 13(5):651-658

31. Park IJ, Kim HC, Yu CS, Kim PN, Won HJ, Kim JC (2008) Radiofrequency ablation for metachronous liver metastases from colorectal cancer after curative surgery. Ann Surg Oncol 15:227232

32. Rubbia-Brandt L, Audard V, Sartoretti P, Roth AD, Brezault C, Le Charpentier M et al (2004) Severe hepatic sinusoidal obstruction associated with oxaliplatin-based chemotherapy in patients with metastatic colorectal cancer. Ann Oncol 15(3):460-466

33. Karoui M, Penna C, Amin-Hashem M, Mitry E, Benoist S, Franc $\mathrm{B}$ et al (2006) Influence of preoperative chemotherapy on the risk of major hepatectomy for colorectal liver metastases. Ann Surg 243(1): $1-7$

34. Vauthey JN, Pawlik TM, Ribero D, Wu TT, Zorzi D, Hoff PM et al (2006) Chemotherapy regimen predicts steatohepatitis and an increase in 90-day mortality after surgery for hepatic colorectal metastases. J Clin Oncol 24(13):2065-2072

35. Tournigand C, Andre T, Achille E, Lledo G, Flesh M, MeryMignard D et al (2004) FOLFIRI followed by FOLFOX6 or the reverse sequence in advanced colorectal cancer: a randomized GERCOR study. J Clin Oncol 22(2):229-237

36. Selzner N, Pestalozzi BC, Kadry Z, Selzner M, Wildermuth S, Clavien PA (2006) Downstaging colorectal liver metastases by concomitant unilateral portal vein ligation and selective intraarterial chemotherapy. Br J Surg 93:587-92

37. Sogaard KK, Astrup LB, Vilstrup H, Gronbaek H (2007) Portal vein thrombosis; risk factors, clinical presentation and treatment. BMC Gastroenterology 15:34

38. Makuuchi M, Hashikura Y, Kawasaki S, Tan D, Kosuge T, Takayama $T$ (1993) Personal experience of right anterior segmentectomy (segments V and VIII) for hepatic malignancies. Surgery 114(1):52-58

39. Elias D, Santoro R, Ouellet JF, Osmak L, de Baere T, Roche A (2004) Simultaneous percutaneous right portal vein embolization and left tumour radiofrequency ablation prior to a major right hepatic resection for bilateral colorectal metastases. Hepatogastroenterol 51(60):1788-1791

40. Folprecht G, Grothey A, Alberts S, Raab HR, Koehne CH (2005) Neoadjuvant treatment of unresectable colorectal liver metastases: correlation between tumour response and resection rates. Ann Oncol 16(8):1311-1319

41. Mitry E, Fields ALA, Bleiberg H, Labianca R, Portier G, Tu D et al (2008) Adjuvant chemotherapy after potentially curative resection of metastases from colorectal cancer: a pooled analysis of two randomized trials. J Clin Oncol 26(30):4906-4911

42. Covey AM, Brody LA, Getrajdman GI, Sofocleus CT, Brown KT (2004) Incidence, patterns, and clinical relevance of variant portal vein anatomy. Am J Roentgenol 183(4):1055-1064 